Research Paper

\title{
Capivasertib restricts SARS-CoV-2 cellular entry: a potential clinical application for COVID-19
}

\author{
Fang Sun ${ }^{1 \#, ~ C h e n g l i n ~} \mathrm{Mu}^{2 \#}$, Hang Fai Kwok ${ }^{3 \#}$, Jiyuan $\mathrm{Xu}^{1}$, Yingliang $\mathrm{Wu}^{1}$, Wanhong Liu ${ }^{4}$, Jean-Marc \\ Sabatier ${ }^{5}$, Cédric Annweiler ${ }^{6}$, Xugang Li ${ }^{\circledR}$, Zhijian $\mathrm{Cao}^{1 \bowtie}$ and Yingqiu Xie ${ }^{{ }^{\boxplus}}$ \\ 1. State Key Laboratory of Virology and Modern Virology Research Center, College of Life Sciences, Wuhan University, Wuhan, 430072, China. \\ 2. Sino German Joint Research Center for Agricultural Biology, and State Key Laboratory of Crop Biology, College of Life Sciences, Shandong Agricultural University, \\ Tai'an, 271018, China. \\ 3. Institute of Translational Medicine, Faculty of Health Sciences, University of Macau, Avenida de Universidade, Taipa, Macau SAR; MoE Frontiers Science Center for \\ Precision Oncology, University of Macau, Avenida de Universidade, Taipa, Macau SAR. \\ 4. Hubei Province Key Laboratory of Allergy and Immunology, School of Basic Medical Sciences, Wuhan University, Wuhan 430071, China. \\ 5. Aix-Marseille University, Institute of NeuroPhysiopathology, UMR 7051, 27, Bd Jean Moulin, 13385 Marseille cedex, France. \\ 6. Department of Geriatric Medicine and Memory Clinic, Research Center on Autonomy and Longevity, University Hospital, Angers, France. \\ 7. School of Sciences and Humanities, Biology Department, and Pilot Cluster of Multidisciplinary Comprehensive Materia Medica, Biocluster within Cluster of Life \\ Science and Engineering at C4, Nazarbayev University, Nur-Sultan, 010000, Republic of Kazakhstan. \\ \#Co-first authors.
}

$\triangle$ Corresponding authors: Yingqiu Xie, 53 Kabanbay Batyr Ave, Nur-Sultan, 010000, Republic of Kazakhstan; Tel: +7 (7172) 694686; Fax: +7 (7172) 694686; E-mail: xieautumnus@yahoo.com; Zhijian Cao, 299 Bayi Road, Wuchang District, Wuhan City, Hubei Province, China; Tel: +86-27-68752831; Fax: +86-27-68756746; E-mail: zjcao@whu.edu.cn; Xugang Li, 61 Daizong Street, Tai'an, 271018, China; Tel: +86-0538-8247590; Fax: +86-0538-8247590; E-mail: xgli@sdau.edu.cn.

(C) The author(s). This is an open access article distributed under the terms of the Creative Commons Attribution License (https://creativecommons.org/licenses/by/4.0/). See http://ivyspring.com/terms for full terms and conditions.

Received: 2021.01.04; Accepted: 2021.05.21; Published: 2021.06.11

\begin{abstract}
Coronavirus disease 2019 (COVID-19) caused by severe acute respiratory syndrome coronavirus 2 (SARS-CoV-2) infection has led to more than 150 million infections and about 3.1 million deaths up to date. Currently, drugs screened are urgently aiming to block the infection of SARS-CoV-2. Here, we explored the interaction networks of kinase and COVID-19 crosstalk, and identified phosphoinositide 3-kinase (PI3K)/AKT pathway as the most important kinase signal pathway involving COVID-19. Further, we found a PI3K/AKT signal pathway inhibitor capivasertib restricted the entry of SARS-CoV-2 into cells under non-cytotoxic concentrations. Lastly, the signal axis PI3K/AKT/FYVE finger-containing phosphoinositide kinase (PIKfyve)/Ptdlns(3,5)P2 was revealed to play a key role during the cellular entry of viruses including SARS-CoV-2, possibly providing potential antiviral targets. Altogether, our study suggests that the PI3K/AKT kinase inhibitor drugs may be a promising anti-SARS-CoV-2 strategy for clinical application, especially for managing cancer patients with COVID-19 in the pandemic era.
\end{abstract}

Key words: COVID-19; SARS-CoV-2; AKT inhibitor; capivasertib; antiviral activity

\section{Introduction}

Pandemic coronavirus disease-2019 (COVID-19) has caused more than 150 million infections and about 3.1 million deaths up to date (https://covid19.who. int/). Although the detailed pathology and molecular mechanisms of COVID-19 are not completely clear yet, the virology investigation provided some knowledge and potential treatment avenues. The virus which causes COVID-19, named as severe acute respiratory syndrome coronavirus 2 (SARS-CoV-2), first enters into host cells by binding to the cellular receptor angiotensin-converting enzyme 2 (ACE2) via receptor-binding domain $(\mathrm{RBD})$ interacting with the
C-terminal domain of SARS-CoV-2 spike protein (S) [1]. The binding is followed by SARS-CoV-2 $S$ priming regulated by transmembrane protease serine 2 (TMPRSS2), which is a key factor for viral entry [1].

Upon SARS-CoV-2 virions attachment to the host cells, viral spike protein shows a conformation change which mediates the viral envelope fusion with the host cell membrane via an endosomal pathway [2]. Targeting viral entry has been proposed and clinical trial has started (https://clinicaltrials.gov/ ct2/show/NCT04352400) [3]. However, the main consequence is the cytokine storm of the host cells 
upon response to infection and followed by signalling pathways transduction to the downstream targets of cell death, cell cycle and others, which may crosstalk with cancer signaling as the similar common interleukin activators [4]. For example, some cytokines transduce signals through JAKs and signal transducer and activator of transcriptions (STATs) whose mislocalization or super-activation may induce cancers [5]. In details, binding of interleukin to their receptors resulting in conformational changes of receptors will provide docking site for recruiting a number of signaling proteins, which may also crosstalk with kinases, such as Pim-1, Ras or AKT activation [6]. In addition, other factors involved in the inflammation responses can also activate cancerrelated cell proliferation signaling [7]. Therefore, kinases most likely involve in the cytokine signaling of COVID-19 inflammation.

Given the similarity of signaling of cancer and COVID-19, a recent study revealed a large scale kinases mediated global phosphorylation of both viral and host cell proteins in a cell line of monkey kidney Vero E6 cells by whole proteomics analysis upon SARS-CoV-2 infection [8, 9] with cytokine storm, activating of kinases including p38 MAP kinase, CDKs, casein kinase II (CK2), and PIKFYVE kinases suggesting the potential clinical applicable kinase inhibitor drugs for the treatment of COVID-19 [8]. Based on different cohort of preclinical and clinical studies, it is urgently needed to analyze whole big data to get a systematic conclusion or prediction of new drugs for cancer patients infected with COVID-19. Here, we aimed to use network pharmacology tool to analyze the database of big data and explore the crosstalk pathways between whole kinases signaling and COVID-19, then to test the efficacy of the predicted drugs in a SARS-CoV-2 model system.

\section{Materials and Methods}

\section{Cells}

Vero cells were purchased from the China Center for Type Culture Collection (CCTCC) and were cultured in DMEM (Gibco-Invitrogen, New York, NY, USA) supplemented with 10\% FBS (GibcoInvitrogen) and $1 \%$ penicillin/streptomycin at $37^{\circ} \mathrm{C}$ in an incubator filled with $5 \% \mathrm{CO}_{2}$.

\section{Construction of protein-protein interaction (PPI) crosstalk-networks between kinase, drug target and COVID-19}

The drug targets of COVID-19 and kinases related targets were collected by searching databases of NCBI (https://www.ncbi.nlm.nih.gov), GenCLiP3
GeneCards, using key words above and "Homo sapiens" for human species plus the input of targets from recent published pool [8] followed by the application of venny 2.1.0 tool to explore of the intersection targets, of which were exported to the STRING database to construct protein-protein networks for further analysis and visualization through Cytoscape 3.7.2 tool [10-13] (http://www. bioinformatics.com.cn). For capivasertib (AZD5363) based drug targets-COVID-19 PPI, the targets were identified based on the drug bank by dissociation constant $(\mathrm{Kd})$ of $100 \mathrm{nM}$ to $10 \mu \mathrm{M}$ range of dose (https://lincs.hms.harvard.edu/db/sm/10510-101-

$1 /)$, and PPIs were established after screening of crosstalk intersection targets by venny 2.1.0 tool with STRING database and through Cytoscape 3.7.2 tool as mentioned above.

\section{Analysis of kyoto encyclopedia of genes and genomes (KEGG) pathways and gene ontology (GO)}

Target genes above were input to DAVID database and KEGG pathway analysis website for analysis of pathways by a threshold $\mathrm{P}<0.01$ and online mapping was applied for visualization.

\section{Preparation of SARS-CoV-2 S pseudovirion and analysis of virus entry}

Vesicular stomatitis virus (VSV) glycoprotein deficient VSV exogenously expressing firefly luciferase (VSV-dG-Luc) and SARS-CoV-2 pseudotyped virus whose $S$ protein was packaged using the VSV-dG-Luc were kindly offered by Professor Huan Yan (State Key Laboratory of Virology, Wuhan University, China). VSV-dG-Luc and SARS-CoV-2 S pseudovirion were used to transduce Vero cells at a multiplicity of infection (MOI) of 1 seeded into 96-well plates. After overnight incubation with the different concentrations of a phosphoinositide 3-kinase (PI3K) / AKT inhibitor capivasertib (Beyotime Biotechnology), the firefly luciferase activity was measured by Dual Luciferase Reporter Assay Kit (DL101-01, Vazyme). Each concentration of capivasertib had 3-5 duplicates. The firefly luciferase activity in the control group without capivasertib was normalized to 1 . Then, the relative luciferase activity in the group with capivasertib $(0.1 \mu \mathrm{M}, 1 \mu \mathrm{M}$ and 10 $\mu \mathrm{M})$ was calculated and drawed. The experiment was repeated at least three times.

\section{Target gene expression with cancer patient overall survival data-based analysis for prognosis}

The database re-analysis based on the transcripts per kilobase million (TPM) was performed for patient 
overall survival according to instructions as described in the website [14]. The poor outcomes of prognosis were identified and non-related outcomes were not further analyzed.

\section{Results}

\section{Network pharmacology analysis identified AKT signaling as potential top ranked kinase crosstalk with COVID-19 pathways}

Firstly, we searched a variety of databases for "COVID-19" and "kinase" targets from NCBI (https://www.ncbi.nlm.nih.gov), GenCLiP3 (http:// ci.smu.edu.cn/genclip3/GeneAssociation.php), Gene Cards (https:/ / www.genecards.org/), databases, and Venny 2.1.0 (https://bioinfogp.cnb.csic.es/tools/ venny/) tool for mapping the common targets. We obtained the predicted encoding intersection targets suggesting the interaction proteins involved in kinase mediated COVID-19 infection, progression, and host cell reaction (Figure 1A). Using the screened 1539 co-targets (18\%), we then established the protein-protein interaction map and analyzed the most essential pathways by nodes, GO and KEGG of top ranking of gene counts (Figure 1C and Figure 2). The top ranked kinases are consistent with the recent publication, such as CDK, p38/MAPK kinases serving as the positive control (Figure 1B) [8]. The new finding is that PI3K/AKT kinase is in the top ranked kinase list (Figure 1B). Moreover, the GO and KEGG data showed that top ranked pathways are involving the cytokine, cell cycle, cancer, mRNA processing, and
A

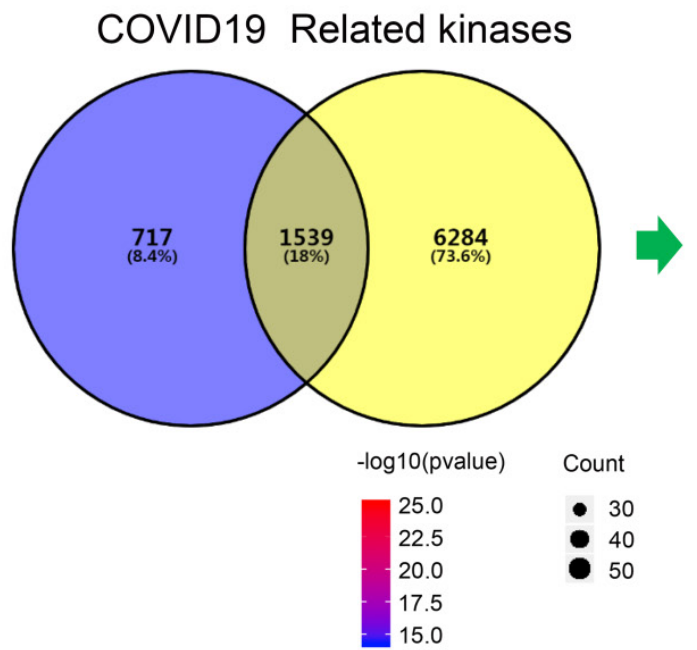

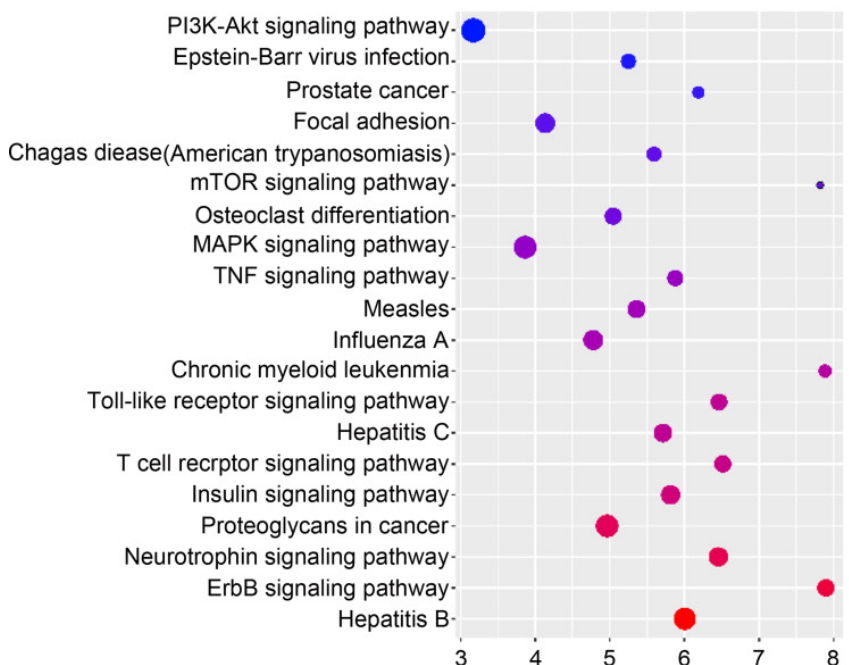

B

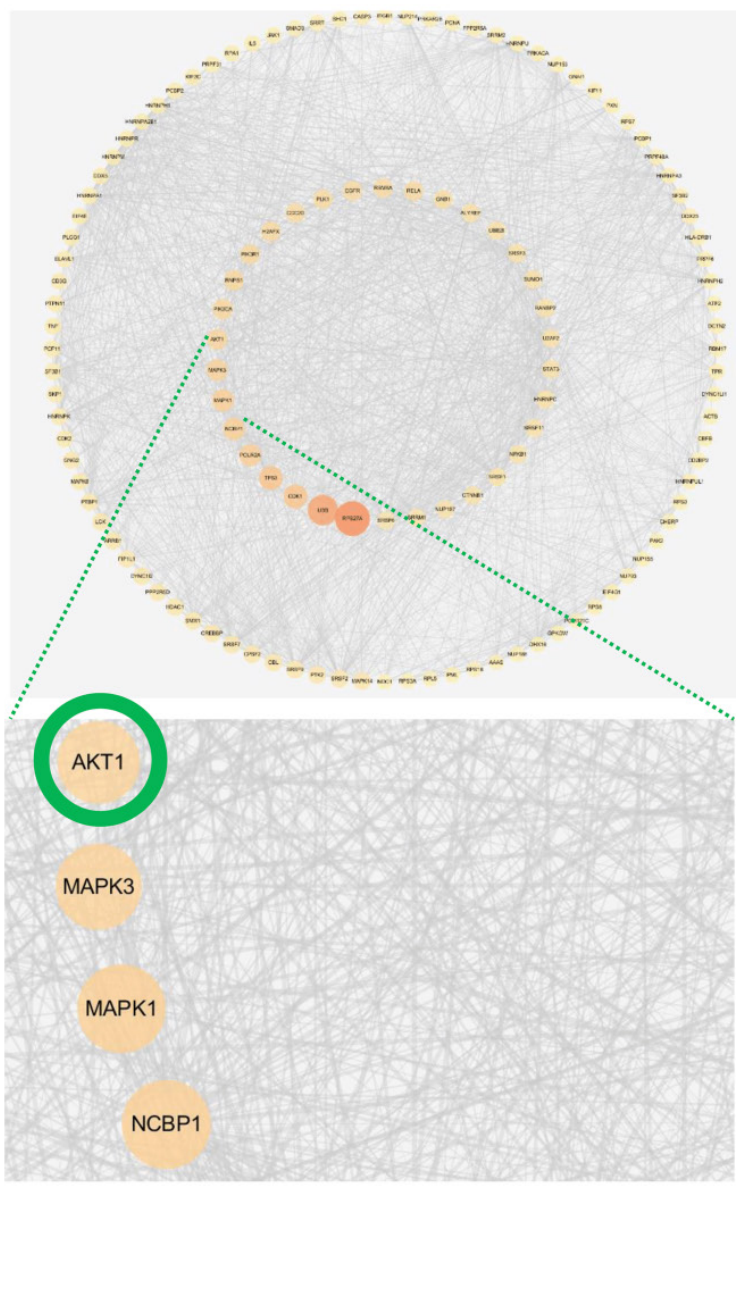

Figure 1. Network pharmacology identified the kinase signaling crosstalk with the COVID-19. A. Analysis of the intersection target genes by venny 2.1.0 tool. The Venn diagram represents the number of intersection of the COVID-19 target and the kinase-related genes. In order to distinguish the two sets of data, different colors are used as the standard. The size of the circle does not represent the proportion, but the proportion of different parts. The purpose is to show the proportion of the data set occupied by the intersection genes. To show the intersection result more clearly, we have modified the color of the text on the graph. B. Establishment protein-protein interaction (PPI) crosstalk-networks between kinase and COVID-19 were constructed as described in the methods. The network of the interaction score was set to 0.9 for screening, and 1244 nodes and 12426 edges were obtained. The average degree value is 20 , and two times the average degree value is selected as the screening condition. The points with a degree value $\geq 40$ are used to visualize the protein interaction network with cytoscape. The size of the node, and the intensity of color, shows the degree of value. Each edge represents the interaction between proteins. The line number indicates the degree of association. C. Kyoto encyclopedia of genes and genomes (KEGG) pathways indicating AKT pathway is the top ranked. 
viral life cycle (Figure $1 \mathrm{C}$ and Figure 2). Thus, our network pharmacology analysis suggests that AKT may be the potential kinases associated with the COVID-19 disease.

\section{AKT inhibition restricts SARS-CoV-2 entry}

Next, we aimed to explore the influence of AKT kinases signaling on COVID-19 by testing the efficacy of the predicted drug in a SARS-CoV-2 model system.
Capivasertib is a potent pan-AKT kinase inhibitor drug that inhibits AKT1, AKT2 and AKT3. Capivasertib had significant antitumor activities, and was being used as an oral small-molecule AKT inhibitor for drug-resistant breast cancer in clinical trials $[15,16]$. Here, we explored the influence of capivasertib on SARS-CoV-2 S protein pseudotyped virus and VSV-dG in Vero cells by the luciferase report assay. The negative-strand genome models of

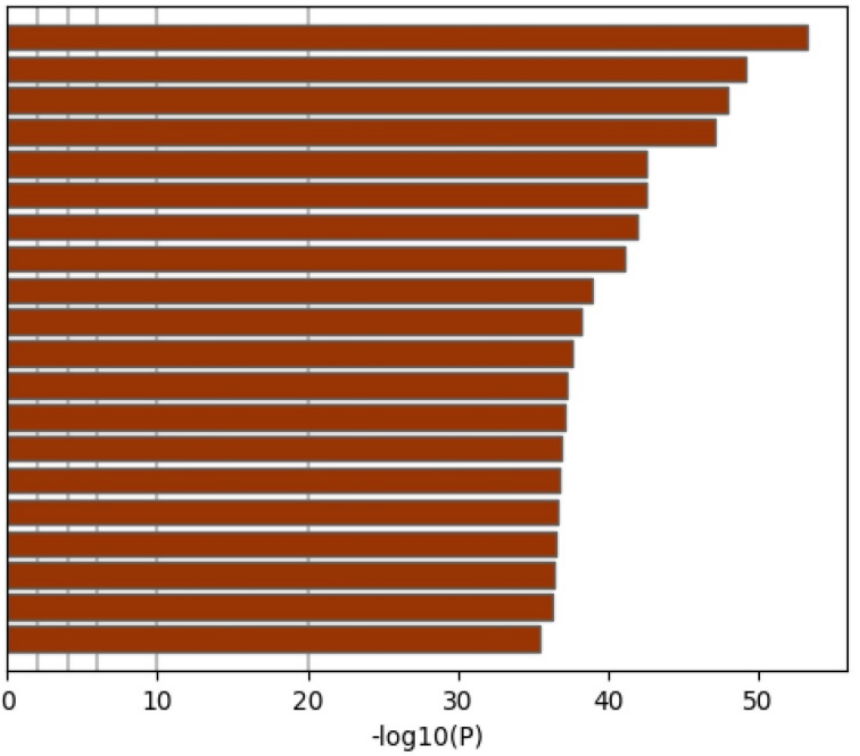

R-HSA-1640170: cell cycle R-HSA-449147:signaling by Interleukins

GO-0051347:positive regulation of transferase activity GO-0007346:regulation of mitotic cell cycle GO-0051169:nuclear transport GO-0006397:mRNA processing GO-1903827:regulation of cellular protein localization GO-0097190:apoptotic signaling pathway GO-0010638:positive regulation of organelle organization R-HSA-2262752:cellular responses to stress GO-0034329:cell iunction assembly GO-0032870:cellular response to hormone stimulus hsa05200:pathways in cancer GO-0051301:cell division GO-0018105:peptidyl-serine phosphorylation GO-0019058:viral life cycle GO-0043065:positive regulation of apoptotic process GO-0034655:nucleobase-containing compound catabolic process GO-0051345:positive regulation of hydrolase activity R-HSA-5663202:diseases of signal transduction by growth factor receptors and second messengers

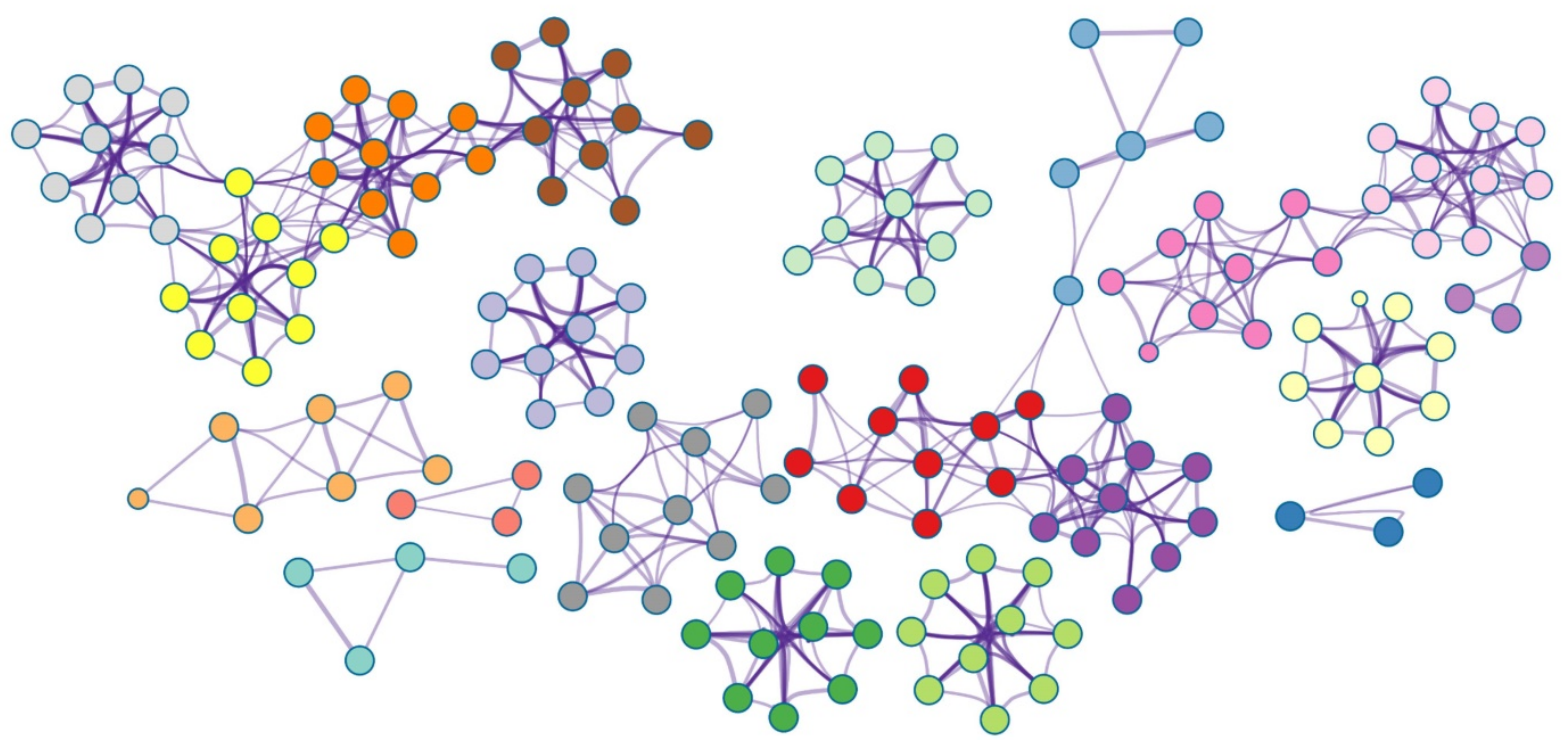

cell cycle

signaling by interleukins

positive regulation or transferase activity

regulation of mitotic cell cycle

nuclear transport

mRNA processing

regulation of cellular protein localization

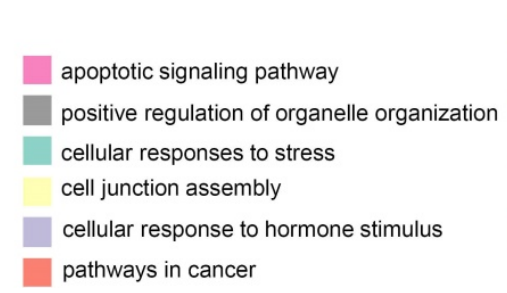

cell division

peptidyl-serine phosphorylation viral life cycle positive regulation of apoptotic process nucleobase-containing compound catabolic process positive regulation of hydrolase activity

diseases of signal transduction by growth factor receptor

Figure 2. Analysis of network related biological functions. Identified statistically enriched items were clustered as a tree by Kappa-statistical similarities and 0.3 kappa score of the threshold was used for clusters followed by converting into network small maps with colors indicating groups. The network was shown with Cytoscape (v3.1.2) and items description indicated. 
VSV, VSV-dG-Luc, and SARS-CoV-2 S pseudovirion were shown in Figure 3A. The quantification analysis showed that capivasertib had a concentrationdependently inhibitory activity against intracellular luciferase activity of SARS-CoV-2 S pseudovirion and the $50 \%$ inhibitory concentration was $2.33 \pm 1.40 \mu \mathrm{M}$ (Figure 3B), while capivasertib $(0.1,1$ and $10 \mu \mathrm{M})$ had no inhibitory activity on the infection of VSV-dG-Luc (Figure 3C). In addition, the treatment of capivasertib $(0.1,1$ and $10 \mu \mathrm{M})$ did almost not affect the growth and survival of Vero cells, but $20 \mu \mathrm{M}$ capivasertib can inhibit 20\% Vero cells (Figure 3D). The 50\% cytotoxic concentration of capivasertib to Vero cells was $82.1 \pm$ $1.52 \mu \mathrm{M}$. The selection index of capivasertib was about 35 , which indicated that capivasertib was relatively safe in vitro. In fact, luciferase activity could reflect viral infection rate changes from two steps of viral entry and viral genome replication/expression. In order to exclude the role of capivasertib during the step of viral genome replication/expression, we tested the effect of capivasertib on VSV-dG-Luc in Vero cells where SARS-CoV-2 spike was overexpressed or not. The data indicated that the treatment of capivasertib had no effect on the luciferase activity of VSV-dG-Luc infecting Vero cells with SARS-CoV-2 spike overexpression or not, suggesting that capivasertib was not related to the genome replication and expression of VSV-dG-Luc (Figure 4). Clearly, these results suggest that capivasertib, an AKT-targeted anti-cancer drug, possibly restricts the entry of SARS-CoV-2 to cells under non-cytotoxic concentrations and has a great potential for clinical trial of anti-SARS-CoV-2.
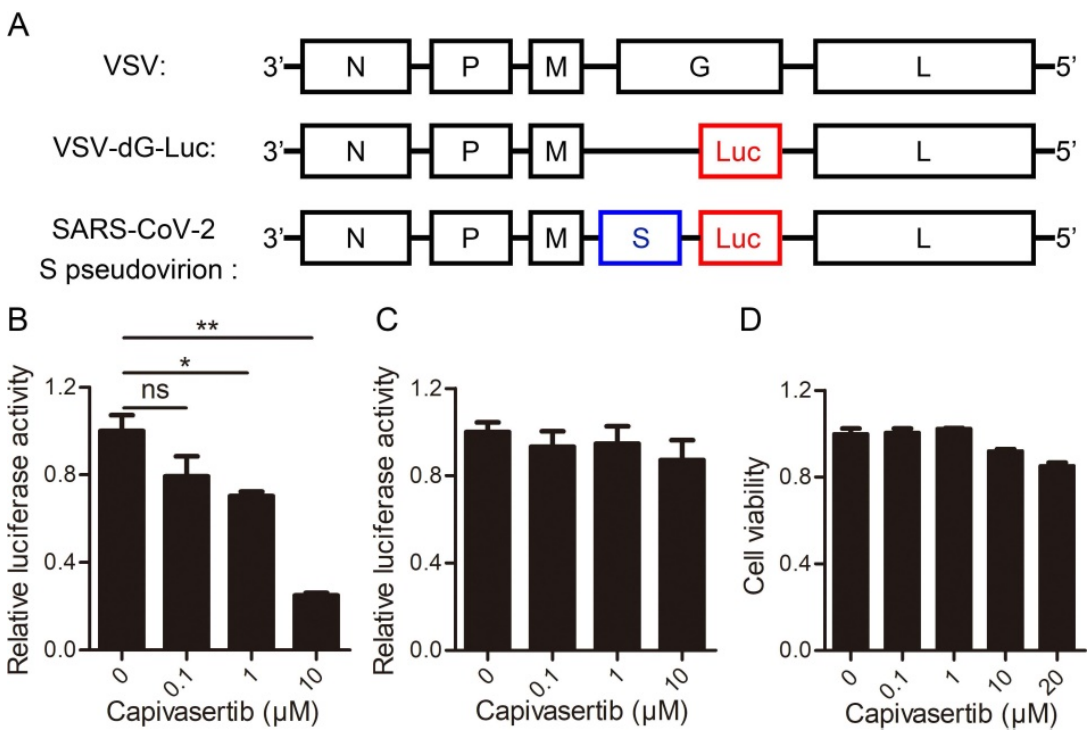

D

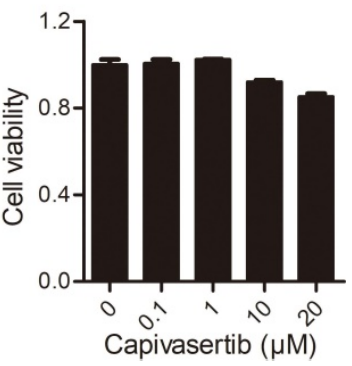

Figure 3. Inhibitory effects of AKT signal inhibitor on the entry of SARS-CoV-2 to cells with a dose-dependent manner under non-cytotoxic concentrations. A. The negative-strand genome model of VSV, VSV-dG-Luc, and SARS-CoV-2 S pseudovirion. Black boxes represent five genes of VSV, which encode nuclear protein $(\mathrm{N})$, phosphate protein $(\mathrm{P})$, matrix protein $(\mathrm{M})$, glycoprotein protein $(\mathrm{G})$, and RNA polymerase $(\mathrm{L})$, respectively. Red box represents firefly luciferase gene, and blue box represents glycoprotein gene of SARS-CoV-2. B. and C. Concentration-dependent inhibition of capivasertib on SARS-CoV-2 entry into Vero cells. Vero cells infected by SARS-CoV-2 S pseudovirion (B) and VSV-dG-Luc (C) at an MOI of 1 were incubated with different concentrations of capivasertib (0, $0.1 \mu M, 1 \mu M$, and $10 \mu M)$. After $20 \mathrm{~h}$, cells were collected and intracellular luciferase activity was analyzed by luciferase reporter assay. D. Cytotoxicity of capivasertib to Vero cells by the MTT assay. ns, no significance. $*, p<0.05$. **, $p<0.01$
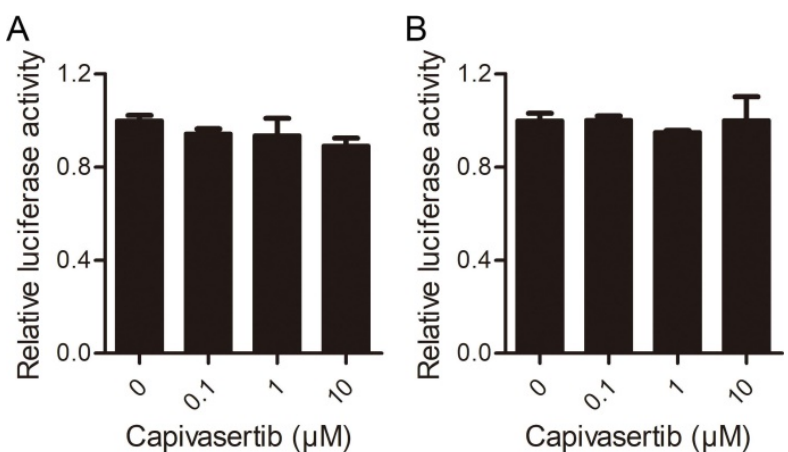

Figure 4. Effects of capivasertib on VSV-dG-Luc in Vero cells with SARS-CoV-2 spike overexpression or not. A. and B. Control vector pCAGGS (A) and the recombinant vector PCAGGS-HA-spike (B) were transfected into Vero cells for $24 \mathrm{~h}$, respectively. Then, cells infected by VSV-dG-Luc at an MOI of 1 were incubated with different concentrations of capivasertib $(0,0.1 \mu \mathrm{M}, 1 \mu \mathrm{M}$, and $10 \mu \mathrm{M})$, respectively. After $20 \mathrm{~h}$, cells were collected and intracellular luciferase activity was analyzed by luciferase reporter assay. 
A

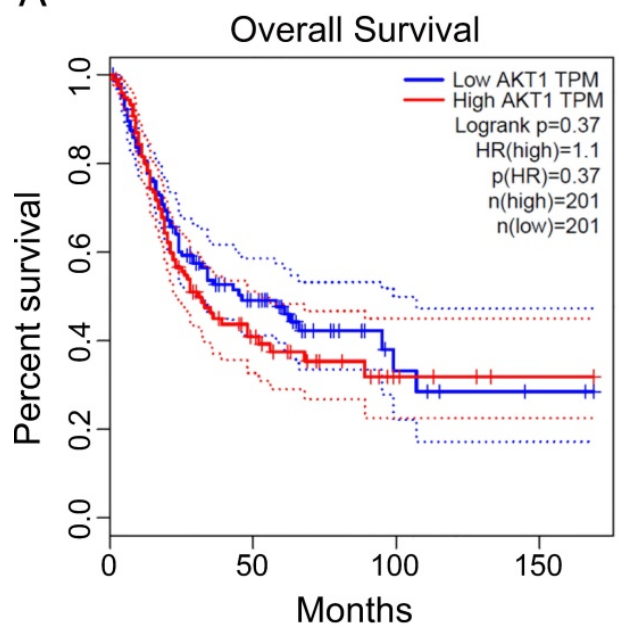

C

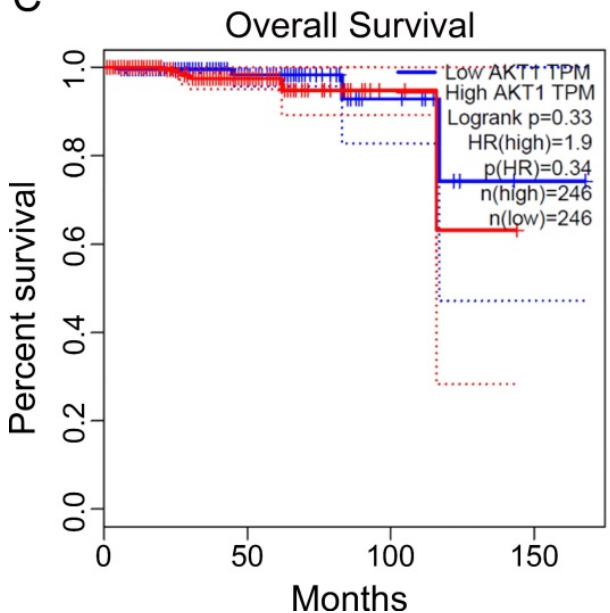

B

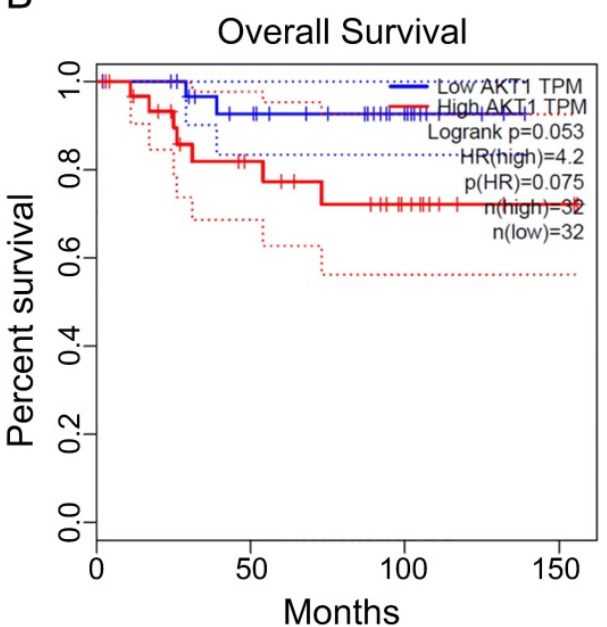

D

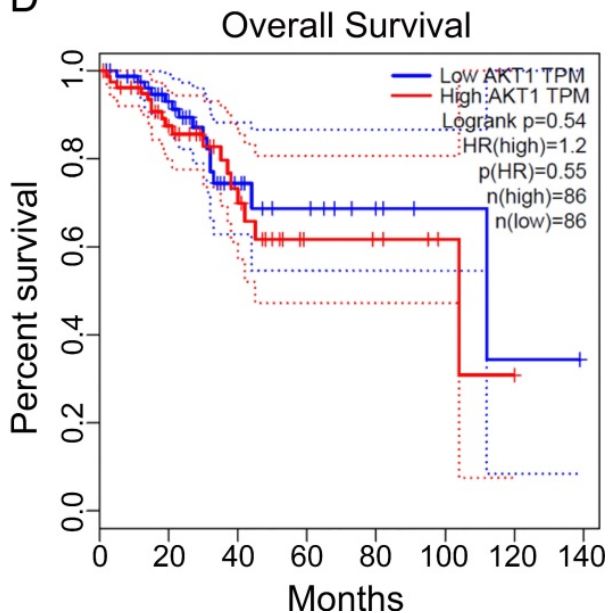

Figure 5. The prognosis of the identified crosstalk targets in cancers. The transcripts per kilobase million (TPM) of specific genes listed were analyzed for patient survival in different cancers. The cancer types are from A to D: urothelial bladder carcinoma, kidney chromophobe carcinoma, prostate adenocarcinoma, and uterine corpus endometrial carcinoma. The data were obtained as described in methods using GEPIA online analysis tool [20] for AKTI prognostic analysis.

\section{AKT profiling in cancer overall survival}

Finally, we searched database of gene expression profiling of the targets and listed the poor outcome of overall survival which correlated to the gene overexpression encoding for AKT kinases. The significant cancer types are urothelial bladder carcinoma, kidney chromophobe carcinoma, prostate adenocarcinoma, uterine corpus endometrial carcinoma for AKT (Figure 5). Thus, our data suggest the identified kinase network related COVID-19 targets has poor prognosis in some types of cancer. The AKT inhibitor drugs, such as capivasertib, could be recommended for concurrent targeting both COVID-19 and the type of cancer in the pandemic era for unique treatment.

\section{Discussion}

Here, we reported a whole kinase-proteinprotein interaction network, which identified a top ranked AKT kinase-mediated pathway and the related clinical anti-cancer drugs for possibly treating COVID-19 patients by targeting AKT. The clinical applicable drug capivasertib has a great potential for clinical trial of anti-SARS-CoV-2.

It was very interesting to investigate the detailed relationship between PI3K/AKT signal pathway and viral infection. The previous many studies reported that the activation of the PI3K/AKT signaling pathway facilitated viral replication [17, 18], which suggested that our finding about the inhibitor of PI3K/AKT restricting SARS-CoV-2 entry to cells was not just one case. The PI3K/AKT signal pathway possibly played a common role during many viral infections. Very importantly, PI3K(PIK3CA)/AKT cascade reaction can activate a FYVE fingercontaining phosphoinositide kinase (PIKfyve), whose principal enzymatic activity is to phosphorylate PtdIns3P to PtdIns(3,5)P2 [17, 18]. PIKfyve participates in several aspects of endosome dynamics via PtdIns(3,5)P2 production. In fact, the endocytic pathway is one of the most important ways for 


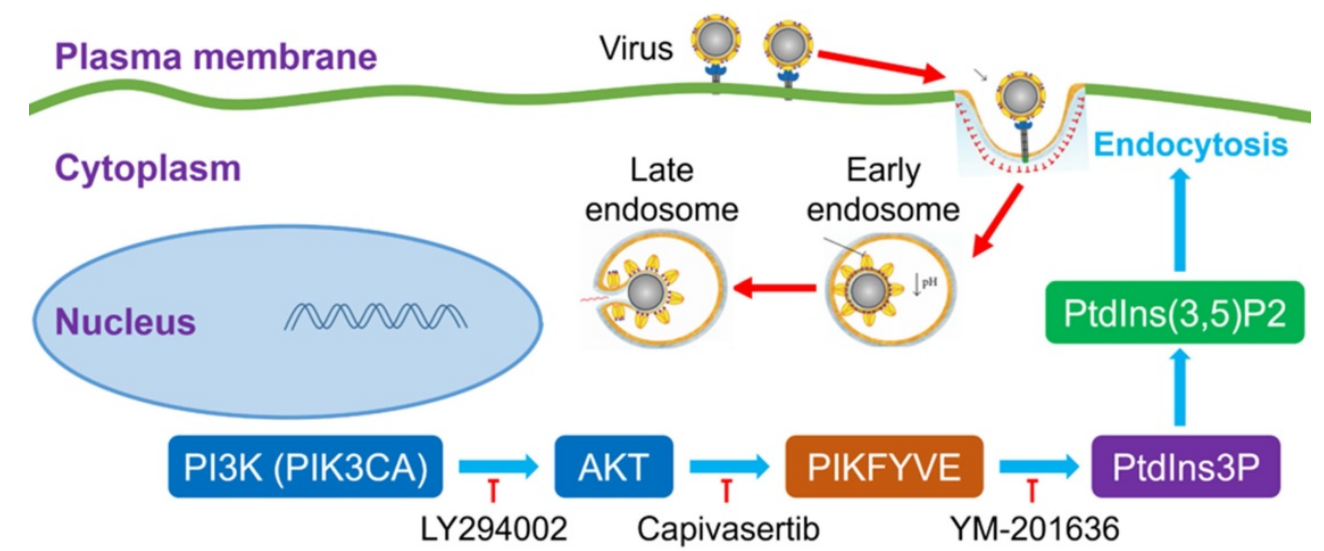

Figure 6. An intracellular pathway model for pharmacological blockage of PI3K(PIK3CA)/AKT signal axis restricting the entry of viruses including SARS-CoV-2. $\longrightarrow$ activation or promotion; $\longmapsto$ inactivation or inhibition; PI3K, phosphatidylinositol 3-kinase; PIK3CA, phosphatidylinositol 3-kinase catalytic subunit $\alpha$; AKT, serine/threonine kinase (PKB, protein kinase B); PIKFYVE, FYVE finger-containing phosphoinositide kinase; Ptdlns3P, phosphatidylinositol-3-phosphate; Ptdlns(3,5)P2, phosphatidylinositol-3,5-biphosphate.

viruses to enter host cells. It was easily believed that the interference of PI3K/AKT/PIKfyve/PtdIns(3,5)P2 signal axis could regulate viral replication. Indeed, a PI3K inhibitor LY294002 was reported to effectively decrease the replication and DNA synthesis of Marek's disease virus (MDV) [17], and a highly selective PIKfyve inhibitor YM-201636 was also found to inhibit retrovirus replication [18]. Therefore, the signal pathway of PI3K/AKT/PIKfyve/PtdIns(3,5)P2 would provide potential druggable targets for many viruses with the endocytic pathway for entering cells (Figure 6).

PI3K/AKT signaling plays a key role in cell proliferation, survival, growth, migration, invasion, and can inhibit apoptosis and promote angiogenesis. Abnormal PI3K/AKT signaling pathway can cause diseases, such as cancer. Cancer has always been one of the major health problems. With the continuous progress of life science, targeted therapy has become the latest popular method for malignant tumor. Among them, inhibitors of the PI3K/AKT pathway play an essential role in cancer treatment. PI3K and AKT are potential tumor drug targets, and their anti-tumor therapies show attractive prospects. Capivasertib is a potent pan-AKT kinase inhibitor drug that inhibits AKT1, AKT2 and AKT3. It had significant antitumor activity and was an attractive lead of antitumor drugs. Capivasertib is being used as an oral small-molecule AKT inhibitor for drug-resistant breast cancer in clinical trials $[19,20$, 21]. Thus, targeting the PI3K/AKT by concurrent anti-cancer and anti-viral drugs would provide potential of the best management of cancer patients in the COVID-19 era.

\section{Conclusion}

Our study showed that a kinase inhibitor capivasertib, also an AKT-targeted anti-cancer drug, could prevent the entry of SARS-CoV-2 to cells. There are so many antitumoral drugs targeting PI3K/AKT signal pathway in clinical trials, which would possibly be valuable drug resources to combat the current COVID-19 pandemic and be worth managing the critical urological cancer patients with COVID-19 in the pandemic era.

\section{Abbreviations}

COVID-19: coronavirus disease-2019; SARS-CoV-2: severe acute respiratory syndrome coronavirus 2; PPI: protein-protein interaction network; ACE2: angiotensin-converting enzyme 2; TMPRSS2: transmembrane protease serine 2; S: spike protein; PI3K: phosphoinositide 3-kinase; RBD: receptor-binding domain; STATs: signal transducer and activator of transcriptions; CK2: casein kinase II; Kd: dissociation constant; KEGG: kyoto encyclopedia of genes and genomes; GO: gene ontology; PIKfyve: FYVE finger-containing phosphoinositide kinase; MDV: Marek's disease virus; TPM: transcripts per kilobase million; VSV: vesicular stomatitis virus; VSV-dG-Luc: glycoprotein deficient VSV exogenously expressing firefly luciferase; MOI: multiplicity of infection.

\section{Acknowledgements}

We thank Professor Huan Yan (State Key Laboratory of Virology, Wuhan University, China) for providing a SARS-CoV-2 spike protein pseudotyped virus system and the recombinant vector pCAGGS-HA-spike.

\section{Funding}

This work was supported by grants from National Science Fund of China (No. 31872239), Fundamental Research Fund for the Central Universities of China (No. 2042021kf0219), Science 
and Technology Development Fund Macau SAR (No. 0055/2019/A1), Hubei Science Fund for Excellent Scholars (No. 2020CFA015), and Nazarbayev University Faculty-Development Competitive Research Grants Program ID: 15798117 (110119FD4531) and ID: 15874919 (110119FD4542).

\section{Author Contributions}

The experiment was designed/performed by Fang Sun, Chenglin $\mathrm{Mu}$, Hang Fai Kwok, Jiyuan $\mathrm{Xu}$, Yingliang $\mathrm{Wu}$, Wanhong Liu, Jean-Marc Sabatier, Cédric Annweiler, Xugang Li, Zhijian $\mathrm{Cao}$ and Yingqiu Xie. The manuscript was written by Fang Sun, Chenglin $\mathrm{Mu}$, Zhijian Cao and Yingqiu Xie. The manuscript was revised and consulted by Yingliang $\mathrm{Wu}$, Jean-Marc Sabatier, Cédric Annweiler, and Hang Fai Kwok.

\section{Competing Interests}

The authors have declared that no competing interest exists.

\section{References}

1. Hoffmann M, Kleine-Weber $\mathrm{H}$, Schroeder $\mathrm{S}$, Kruger N, Herrler $\mathrm{T}$, Erichsen $\mathrm{S}$, et al. SARS-CoV-2 Cell Entry Depends on ACE2 and TMPRSS2 and Is Blocked by a Clinically Proven Protease Inhibitor. Cell. 2020; 181: 271-80 e8.

2. Shereen MA, Khan S, Kazmi A, Bashir N, Siddique R. COVID-19 infection: Origin, transmission, and characteristics of human coronaviruses. J Adv Res. 2020; 24: 91-98.

3. Kwok HF. Review of Covid-19 vaccine clinical trials - A puzzle with missing pieces. International Journal of Biological Sciences. 2021; 17: 1461-1468.

4. Mangalmurti N, Hunter CA. Cytokine Storms: Understanding COVID-19. Immunity. 2020; 53: 19-25.

5. Lee M, Rhee I. Cytokine signaling in tumor progression. Immune Netw. 2017; 17: 214-227.

6. Kim O, Jiang T, Xie Y, Guo Z, Chen H, Qiu Y. Synergism of cytoplasmic kinases in IL6-induced ligand-independent activation of androgen receptor in prostate cancer cells. Oncogene. 2004; 23: 1838-1844.

7. Astuti I, Ysrafil. Severe Acute Respiratory Syndrome Coronavirus 2 (SARSCoV-2): An overview of viral structure and host response. Diabetes Metab Syndr. 2020; 14: 407-412.

8. Song H, Seddighzadeh B, Cooperberg MR, Huang FW. Expression of ACE2, the SARS-CoV-2 receptor, and TMPRSS2 in prostate epithelial cells. bioRxiv. 2020.

9. Case JB, Bailey AL, Kim AS, Chen RE, Diamond MS. Growth, detection, quantification, and inactivation of SARS-CoV-2. Virology. 2020; 548: 39-48.

10. Jia-Hong Wang L-FZ, Hua-Feng Wang, Yue-Ting Wen, Kui-Kui Jiang, Xiang-Ming Mao, Zi-Ying Zhou, Kai-Tai Yao, Qing-Shan Geng, Dan Guo. GenCLiP 3: mining human genes' functions and regulatory networks from PubMed based on co-occurrences and natural language processing. Bioinformatics. 2020; 36: 1973-1975.

11. Sun L, Dong S, Ge Y, Fonseca JP, Robinson ZT, Mysore KS, et al. DiVenn: An interactive and integrated web-based visualization tool for comparing gene lists. Front Genet. 2019; 10: 421

12. Szklarczyk D, Gable AL, Lyon D, Junge A, Wyder S, Huerta-Cepas J, et al. STRING v11: protein-protein association networks with increased coverage, supporting functional discovery in genome-wide experimental datasets. Nucleic Acids Res. 2019; 47: D607-D613.

13. Paul Shannon AM, Owen Ozier, Nitin S Baliga, Jonathan T Wang, Daniel Ramage, Nada Amin, Benno Schwikowski, Trey Ideker. Cytoscape: a software environment for integrated models of biomolecular interaction networks. Genome Res. 2003; 13: 2498-2504.

14. Tang Z, Li C, Kang B, Gao G, Li C, Zhang Z. GEPIA: a web server for cancer and normal gene expression profiling and interactive analyses. Nucleic Acids Res. 2017; 45: W98-W102.

15. Ludmir EB, McCaw ZR, Kim DH, Tian L, Wei LJ. Fulvestrant plus capivasertib for metastatic breast cancer. The Lancet Oncology. 2020; 21: e233.

16. Kolinsky MP, Rescigno P, Bianchini D, Zafeiriou Z, Mehra N, Mateo J, et al. A phase I dose-escalation study of enzalutamide in combination with the AKT inhibitor AZD5363 (capivasertib) in patients with metastatic castrationresistant prostate cancer. Ann Oncol. 2020; 31: 619-625.

17. Kindrachuk J, Ork B, Hart BJ, Mazur S, Holbrook MR, Frieman MB, et al. Antiviral potential of ERK/MAPK and PI3K/AKT/mTOR signaling modulation for Middle East respiratory syndrome coronavirus infection as identified by temporal kinome analysis. Antimicrob Agents Chemother. 2015; 59: 1088-1099.

18. Ikonomov OC, Sbrissa D, Shisheva A. YM201636, an inhibitor of retroviral budding and PIKfyve-catalyzed PtdIns(3,5)P2 synthesis, halts glucose entry by insulin in adipocytes. Biochem Biophys Res Commun. 2009; 382: 566-570.

19. Li H, Zhu J, He M, Luo O, Liu F, Chen R. Marek's disease virus activates the PI3K/Akt pathway through interaction of its protein meq with the p85 subunit of PI3K to promote viral replication. Front Microbiol. 2018; 9: 2547.

20. Yap TA, Kristeleit R, Michalarea V, Pettitt SJ, Lim JSJ, Carreira S, et al. Phase I trial of the PARP inhibitor olaparib and AKT inhibitor capivasertib in patients with BRCA1/2- and non-BRCA1/2-mutant cancers. Cancer Discov. 2020; 10: 1528-1543.

21. Wei L, Zhu S, Wang J, Liu J. Activation of the phosphatidylinositol 3-kinase/ Akt signaling pathway during porcine circovirus type 2 infection facilitates cell survival and viral replication. J Virol. 2012; 86: 13589-13597. 